Fabrication of Cryogenic Manganite Bolometers to Measure the Total Energy at the LCLS Free Electron X-ray Laser

O. B. Drury, G. J. Yong, R. M. Kolagani, Y. Liang, C. Gardner, E. Ables, K. W. Fong, R. M. Bionta, S. Friedrich

June 24, 2008

Symposium on Radiation Measurements and Applications Berkeley, CA, United States June 2, 2008 through June 5, 2008 
This document was prepared as an account of work sponsored by an agency of the United States government. Neither the United States government nor Lawrence Livermore National Security, LLC, nor any of their employees makes any warranty, expressed or implied, or assumes any legal liability or responsibility for the accuracy, completeness, or usefulness of any information, apparatus, product, or process disclosed, or represents that its use would not infringe privately owned rights. Reference herein to any specific commercial product, process, or service by trade name, trademark, manufacturer, or otherwise does not necessarily constitute or imply its endorsement, recommendation, or favoring by the United States government or Lawrence Livermore National Security, LLC. The views and opinions of authors expressed herein do not necessarily state or reflect those of the United States government or Lawrence Livermore National Security, LLC, and shall not be used for advertising or product endorsement purposes. 


\title{
Fabrication of Cryogenic Manganite Bolometers to Measure the Total Energy at the LCLS Free Electron X-ray Laser
}

\author{
Owen B. Drury, Grace J. Yong, Rajeswari M. Kolagani, Yong Liang, Christopher S. Gardner, Elden \\ Ables, Kirby W. Fong, Richard M. Bionta, Stephan Friedrich
}

\begin{abstract}
We are developing cryogenic bolometers to measure the total energy of the Linac Coherent Light Source (LCLS) free electron X-ray laser that is currently being built at the Stanford Linear Accelerator Center. LCLS will produce ultrashort $\sim 200$ fs $\mathrm{X}$-ray laser pulses with $\sim 10^{13}$ photons at $0.8 \mathrm{keV}$ up to $\sim 10^{12}$ photons at $8 \mathrm{keV}$ per pulse at a repeat interval as short as $8 \mathrm{~ms}$, and will be accompanied by a halo of spontaneous undulator radiation. Our bolometer consists of a $375 \mu \mathrm{m}$ thick $\mathrm{Si}$ absorber and a $\mathrm{Nd}_{0.67} \mathrm{Sr}_{0.33} \mathrm{MnO}_{3}$ sensor operated at its metal-insulator transition. It will measure the total energy of each pulse with a precision of $<1 \%$, and is designed to meet the conflicting requirements of radiation hardness, sensitivity, linearity over a dynamic range of three orders of magnitude, and readout speed compatible with the LCLS pulse rate. Here we discuss bolometer design and fabrication, and the photoresponse of prototype devices to pulsed optical lasers.
\end{abstract}

Index Terms - Radiation Detectors, Bolometers, Free Electron Lasers, Linac Coherent Light Source, CMR materials, NSMO

\section{INTRODUCTION}

$\mathrm{O}$ VER the next decade, several free-electron X-ray lasers (FELs) will come on line whose peak brightness will exceed that of third generation synchrotrons by ten orders of magnitude. Among them, the Linac Coherent Light Source (LCLS) will be the first to extend the range of operation into the X-ray range from 0.8 to $8 \mathrm{keV}$ [1]. LCLS will pass a pulsed electron beam with an energy of 4 to $14 \mathrm{GeV}$ through a $130 \mathrm{~m}$ long undulator in the last third of the Stanford Linear Accelerator to produce lasing by self-amplified stimulated emission. Lasing occurs if the spontaneous radiation from the first part of the undulator overlaps with the electron pulse and induces a density modulation ("microbunching") that

Manuscript received June 2, 2008. This work was performed under the auspices of the U.S. Department of Energy by Lawrence Livermore National Laboratory under Contract DE-AC52-07NA27344.

O. B. Drury, C. Gardner, E. Ables, K. Fong, R. Bionta and S. Friedrich are with the Applied Physics Division at Lawrence Livermore National Laboratory, Livermore, CA 94550 (phone: +1-925-423-1527; fax: +1-925422-7310; e-mail: friedrich1@1lnl.gov, bionta1@1lnl.gov).

G. Yong and R. M. Kolagani are with the Department of Physics, Geosciences and Astronomy at Towson University, Towson, MD 21252 (email: mrajeswa@towson.edu)

Yong Liang is with Motorola Laboratories, Tempe, AZ 85284 (e-mail: yong.liang@motorola.com) enhances the density and coherence of the emitted radiation [2]. LCLS will produce coherent monochromatic X-ray pulses with $\sim 10^{12}-10^{13}$ photons per pulse at $\sim 200$ fs pulse duration. The laser beam shape will be Gaussian, with the beam diameter varying from $\sim 1 \mathrm{~mm}$ FWHM at $0.8 \mathrm{keV}$ to $\sim 0.2 \mathrm{~mm}$ at $1 \mathrm{keV}$, and will be accompanied by a halo of spontaneous undulator radiation. The pulse rate will initially be $10 \mathrm{~Hz}$, later be increased to $30 \mathrm{~Hz}$, and ultimately to $120 \mathrm{~Hz}$.

A primary concern for beam diagnostics is that the very high power density at LCLS will saturate or damage conventional sensors. LCLS will therefore be equipped with a suite of different detector technologies with complementary characteristics (table 1) [3]. Among them, a thermal detector is thought to contain the least unknowns, since most of the deposited energy will eventually be converted into heat, and be most easily calibratable, since its response will not depend on the details of the pulse duration and the nature of the energy source.

TABLE I

DETECTOR SUITE FOR BEAM CHARACTERIZATION AT LCLS

\begin{tabular}{|c|c|c|}
\hline Technology & Advantage & Disadvantage \\
\hline Gas detector & Indestructible & $\begin{array}{l}\text { Non-linearity / saturation } \\
\text { Calibration }\end{array}$ \\
\hline Scintillator & $\begin{array}{l}\text { Imaging } \\
\text { Sensitivity }\end{array}$ & $\begin{array}{l}\text { Radiation damage } \\
\text { Dynamic range / saturation }\end{array}$ \\
\hline Thermal detector & $\begin{array}{l}\text { Least unknowns } \\
\text { Calibratable }\end{array}$ & $\begin{array}{l}\text { Radiation damage } \\
\text { Sensitivity }\end{array}$ \\
\hline
\end{tabular}

The requirements for the thermal sensor, also referred to as a bolometer or a total energy monitor, are to withstand the impact of the unattenuated LCLS laser beam up to $2 \mathrm{~mJ}$ per $200 \mathrm{fs}$ pulse, and measure the total energy of each pulse with a repeatability $<1 \%$ at $0.2 \mathrm{~mJ}$. It should be sensitive down to the level of the spontaneous background radiation of $1 \mu \mathrm{J}$ per pulse, and be calibratable with an absolute accuracy of $10 \%$ at $0.2 \mathrm{~mJ}$. Since the total energy monitor will likely be used mostly during LCLS start-up to calibrate the other diagnostic sensors, it must be operable at a repeat frequency of $10 \mathrm{~Hz}$, although eventual operation at $120 \mathrm{~Hz}$ is desirable. It should be able to handle beam jitter, expected to be less than $\pm 10 \%$ of the beam diameter, or $\pm 100 \mu \mathrm{m}$ at an FEL energy of $0.8 \mathrm{keV}$. Uncertainty about the initial LCLS characteristics and material response make some degree of flexibility desirable [4]. 


\section{BOLOMETER DESIGN}

\section{A. Design Considerations}

Radiation hardness in the extremely bright LCLS beam restricts the choice of absorber materials to light elements whose X-ray absorption lengths are long and where the deposited energy densities are therefore low. Single crystal absorbers are preferred, since the absorber also needs to serve as the substrate for sensor growth, and epitaxial films, which tend to have lower noise, require single crystal substrates. In addition, the substrate should have a high thermal diffusivity for a pulse decay time compatible with the LCLS repeat interval. Silicon emerges as the absorber material of choice, since it is radiation-hard [5] and there exists a significant literature about thin film deposition on $\mathrm{Si}$ because of its commercial importance. Sapphire $\left(\mathrm{Al}_{2} \mathrm{O}_{3}\right)$ was considered as an alternative, but it is transparent, and sensors on $\mathrm{Al}_{2} \mathrm{O}_{3}$ are therefore difficult to calibrate with optical lasers.

The LCLS X-ray laser energy range from 0.8 to $8 \mathrm{keV}$ sets the minimum thickness of the absorber, in the case of Si to $\sim 400 \mu \mathrm{m}$ for $>99.5 \%$ absorption at $8 \mathrm{keV}$. However, the laser is accompanied by a halo of spontaneous radiation in the higher harmonics of the undulator that extends up to $\sim 400 \mathrm{keV}$. To limit the contribution of this halo to the total signal, the Si should not be significantly larger than necessary.

For flexibility, the sensor material was chosen from the family of rare earth manganites, also known as colossal magnetoresistive (CMR) materials since they exhibit a metalinsulator transition whose temperature changes with magnetic field. While their magnetoresistive properties are irrelevant for bolometer operation, their sharp transition of resistance with temperature is essential, and the tunability of that transition allows mid-term corrections in case unexpected problems arise. These CMR manganites have the general composition $(\mathrm{RE})_{\mathrm{x}}(\mathrm{EA})_{1-\mathrm{x}} \mathrm{MnO}_{3}$, where $\mathrm{RE}$ is a rare-earth and $\mathrm{EA}$ an earthalkaline element. The choice of elements and their ratio $\mathrm{x}$ determines the temperature of the metal-insulator transition, and it can be varied over a wide range by adjusting the material composition $[6,7]$. For low temperature bolometers $\mathrm{Nd}_{\mathrm{x}} \mathrm{Sr}_{(1-\mathrm{x})} \mathrm{MnO}_{3}$ (NSMO) is appropriate [6], with the transition temperature decreasing with increasing Nd-fraction $x$. Sensitivities, typically defined through the temperature coefficient of resistance TCR $\equiv 1 / R \times \partial R / \partial T$, can be around $10 \% / \mathrm{K}$, and square resistances for NSMO vary between $\sim 10$ and $1000 \Omega$ for film thicknesses from $\sim 20$ to $500 \mathrm{~nm}$. Epitaxial CMR films, required to suppress $1 /$ f noise, hysteresis and phase separation, can be grown on Si with the appropriate choice of buffer materials.

Our initial bolometer design was therefore based on an NSMO sensor on a buffered Si substrate with a thickness of $\sim 400 \mu \mathrm{m}$. The sensor is located on the opposite side of the absorption to shield it from the LCLS impact, and the sides of the substrate are clamped to a cryostat for fast pulse decay [4].

\section{B. Finite Element Simulations}

We have performed finite element simulations of the sensor response to calculate the optimum operating temperature and

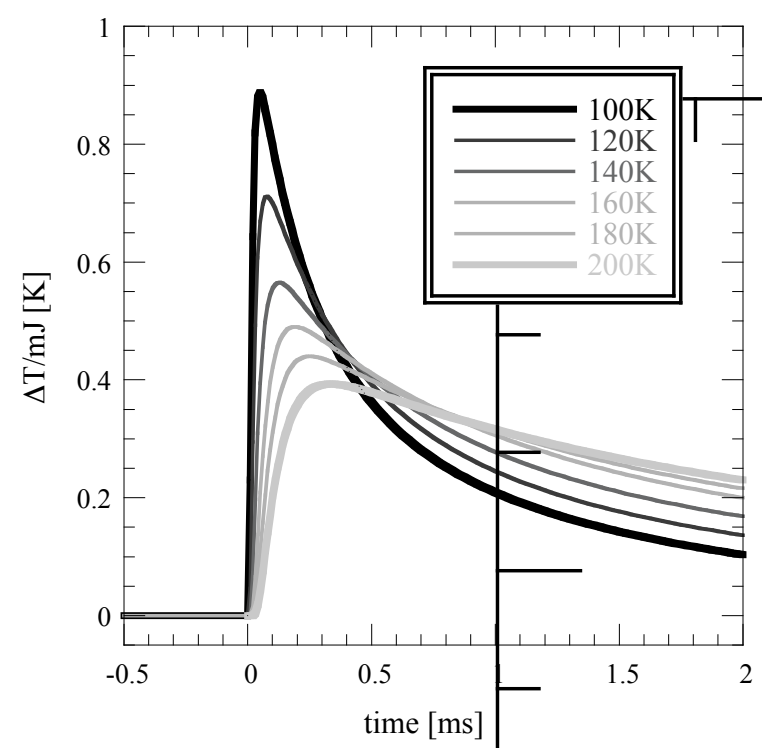

Fig. 1. Finite-element simulation of the thermal response of a $1 \mathrm{~mm} \times 1 \mathrm{~mm}$ sensor on the back of a $370 \mu \mathrm{m}$ thick Si absorber to an LCLS pulse of $1 \mathrm{~mJ}$ at $800 \mathrm{eV}$ as a function of temperature.

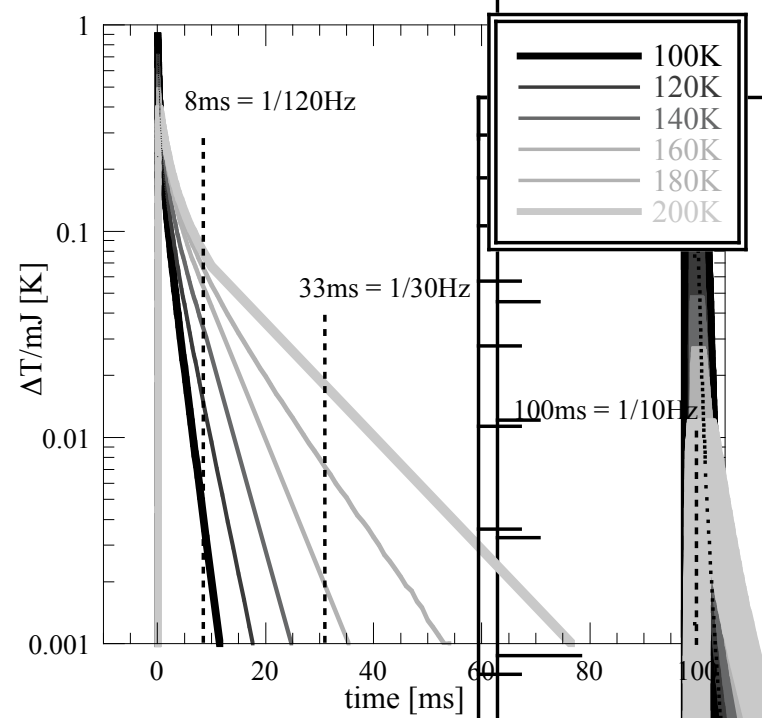

Fig. 2. The same data as in figure 1, but plotted on a logarithmic scale for time scales up to $100 \mathrm{~ms}$. Note that all sensor temperatures $\leq 200 \mathrm{~K}$ are compatible with an LCLS pulse frequency of $10 \mathrm{~Hz}$, but operation at $120 \mathrm{~Hz}$ requires an operating temperature around $\sim 100 \mathrm{~K}$.

device geometry. The simulation uses a $161 \times 241 \times 21$ grid for the $8 \mathrm{~mm} \times 12 \mathrm{~mm} \times 0.37 \mathrm{~mm} \mathrm{Si}$ absorber, with perfect coupling to the cryostat at its two short edges and to the NSMO sensor. The LCLS signal is modeled as a Gaussian with a total energy $E=1 \mathrm{~mJ}$ and an absorption length of $1.5 \mu \mathrm{m}$ corresponding to the absorption length in $\mathrm{Si}$ at the initial LCLS energy of $0.8 \mathrm{keV}$. The temperature evolution is calculated by expanding the heat flow equation to first order, and propagating the temperature of each volume element in the grid according to the temperature differences to its six neighbors. The sensor signal is extracted by averaging the response over the sensor area, taking into account the temperature dependence of the Si heat capacity $\mathrm{C}_{\mathrm{Si}}(\mathrm{T})$ and the $\mathrm{R}(\mathrm{T})$ characteristics of the NSMO sensor. 
Figure 1 shows the simulated response for an operating temperature between 100 and $200 \mathrm{~K}$. Signals are faster and larger at lower operating temperatures because the decreased Si heat capacity $\mathrm{C}_{\mathrm{Si}}(\mathrm{T})$ decreases the rise time $\left(\tau \propto \mathrm{C}_{\mathrm{Si}} / \mathrm{G}\right)$ and increases the temperature excursion $\left(\Delta \mathrm{T} \propto \mathrm{E} / \mathrm{C}_{\mathrm{Si}}\right)$. Amplitudes are of order $1 \mathrm{~K}$ as expected for typical Si heat capacities of $\sim 1 \mathrm{~mJ} / \mathrm{K}$.

The same data are re-plotted in figure 2 on a logarithmic scale with the time axis extending to $100 \mathrm{~ms}$. Assuming that stable biasing at a constant temperature requires that the signal has decayed to at least $\sim 1 \%$, preferably $\sim 0.1 \%$ of its peak value before absorption of the next pulse, all operating temperatures below $\sim 200 \mathrm{~K}$ are compatible with an LCLS repeat frequency of $10 \mathrm{~Hz}$ during commissioning. LCLS characterization at $30 \mathrm{~Hz}$ requires a total energy monitor with a somewhat lower temperature, although it should still be possible for $\mathrm{T} \leq 180 \mathrm{~K}$, provided that the thermal coupling between $\mathrm{Si}$ and the cryostat is good. If in the future operation at $120 \mathrm{~Hz}$ is desired, the NSMO sensor should be designed for an operating temperature around $\sim 100 \mathrm{~K}$.

\section{Device Layout}

We have chosen to put several independent bolometers onto the sensor head that can be moved into the LCLS laser beam path to address concerns about radiation damage and possible non-idealities in the LCLS beam characteristics during the initial start-up. The simplest device consists of a single square $1 \mathrm{~mm} \times 1 \mathrm{~mm}$ NSMO sensor in the center of the back side of the $8 \mathrm{~mm} \times 12 \mathrm{~mm} \mathrm{Si}$ absorber (figure 3a). It has a small area and thus a high sensitivity, because the volume of Si that the FEL energy must heat is small. This comes at the expense of high sensitivity to beam jitter. For moderate beam jitter, we are building single pixel bolometers with larger $2 \mathrm{~mm} \times 2 \mathrm{~mm}$ area, whose response varies less with impact location, as long as the variation is less than the expected $\pm 10 \%$ of the beam diameter, or $\pm 0.1 \mathrm{~mm}$ at $800 \mathrm{eV}$ (figure $3 \mathrm{~b}$ ). These devices have a lower sensitivity because of the larger volume of Si that must be heated beneath the NSMO sensor.
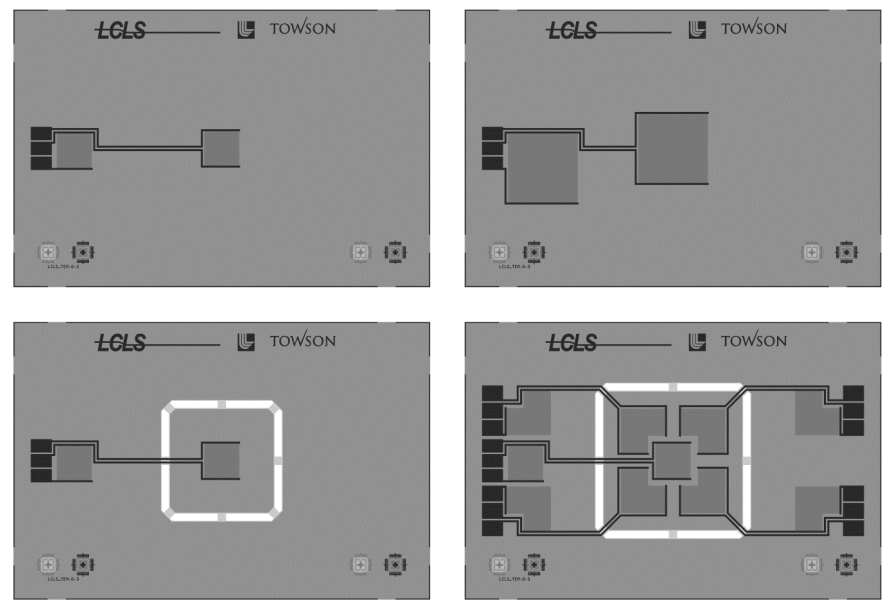

Fig. 3. Mask layout for different types of NSMO bolometers: a) Single small pixel for highest sensitivity and small jitter. b) Single large pixel for moderate jitter. c) Single pixel on Si island d) Five-pixel device for spatial resolution. All sensors have matching bridge completion resistors on the edge of the chip.

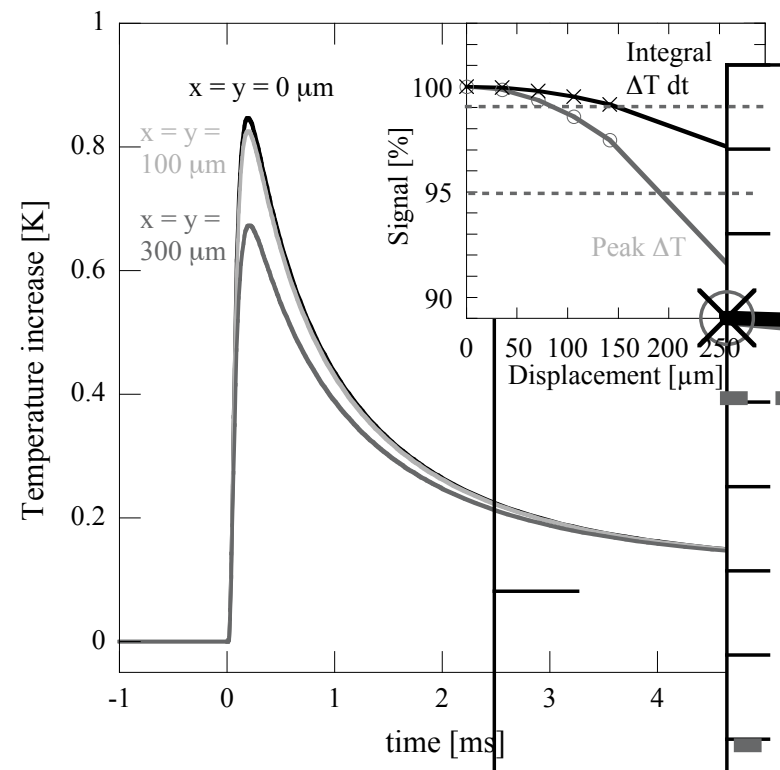

Fig. 4. Simulated response of the "island" devices for different absorption locations displaced from the center $(x=y=0)$ along the island diagonal. The inset demonstrates that the peak of the signal (circles) varies more strongly with absorption location that the integral of the signal (crosses).

For unexpectedly large jitter or beam diameter, we are fabricating devices with channels etched into the Si that leave the bolometer on a free-standing island supported by 8 thin $\mathrm{Si}$ bridges. This "island" design, both as single pixel (figure 3c) and five pixel devices (figure 3d), slows down the response, but is significantly less sensitive to variations in absorption location because the heat spreads out evenly across the island and heats it evenly before it decays to the cryostat temperature across the thin bridges. This effect is especially strong when the integral of the temperature excursion $\int \mathrm{T}(\mathrm{t}) \mathrm{dt}$ rather than the peak of $\mathrm{T}(\mathrm{t})$ is calculated as a measure of the pulse energy (figure 4). The maximum predicted jitter of $10 \%$ of the beam diameter, i.e. $\pm \sim 100 \mu \mathrm{m}$ variation in the absorption location at a photon energy of $800 \mathrm{eV}$, will change the value of the integral to $10 \mathrm{~ms}$ by less than $1 \%$, and jitter three times larger than expected will still change the integral by less than $5 \%$, well within the design specifications (inset figure 4).

\section{PRototype Bolometers FOR $100 \mathrm{~K}$ OPERATION}

\section{A. Bolometer Fabrication}

The chemical reactivity of Si with CMR materials and the easy formation of $\mathrm{SiO}_{2}$ require the use of buffer layers to grow epitaxial NSMO films on $\mathrm{Si}$. We have designed the initial CMR bolometers for operation around $\sim 100 \mathrm{~K}$, using a single $10 \mathrm{~nm}$ strontium titanate $\mathrm{SrTiO}_{3}$ (STO) buffer. The STO is deposited by molecular beam epitaxy at growth temperatures of $400^{\circ} \mathrm{C}$ to $550^{\circ} \mathrm{C}$ at Motorola Laboratories [8]. X-ray diffraction measurements confirm that the STO is epitaxial (figure 5). Note that STO grows $45^{\circ}$ rotated on top of Si, causing the peaks of STO at angles to be displaced by $45^{\circ}$ relative to those of Si peaks.

The NSMO film is deposited by pulsed laser deposition (PLD) at Towson University using a ceramic $\mathrm{Nd}_{0.67} \mathrm{Sr}_{0.33} \mathrm{MnO}_{3}$ 
target and a $\mathrm{KrF}$ excimer laser at a wavelength of $248 \mathrm{~nm}$ and a rate of $5 \mathrm{~Hz}$ [9]. For a fluence of $\sim 2 \mathrm{~J} / \mathrm{cm}^{2}$ on the target, the deposition rate was $\sim 0.03 \mathrm{~nm}$ per pulse, and typical films have a total thickness of $60 \mathrm{~nm}$. During deposition, the substrate is held at a temperature of $770^{\circ} \mathrm{C}$ in an atmosphere of $400 \mathrm{mTorr}$ of $\mathrm{N}_{2} \mathrm{O}$, and it is cooled to room temperature at a rate of $5^{\circ} \mathrm{C} / \mathrm{min}$ in 400 Torr of $\mathrm{O}_{2}$ [9]. This produces epitaxial (figure 5 ) and slightly oxygen-deficient NSMO films, whose peak in the metal insulator transition occurs at lower temperature than the $200 \mathrm{~K}$ typically measured for NSMO films [6].

The sensor dimensions are defined photolithographically with a room temperature acid etch in a solution of potassium iodide (KI) and 10\% hydrochloric acid ( $\mathrm{HCl})$. The $200 \mathrm{~nm} \mathrm{Au}$ wiring layer is deposited by magnetron sputtering, and photolithographically defined by lift-off. To ensure proper adhesion of the $\mathrm{Au}$ to the $\mathrm{Si}$, the Si chip is first cleaned for $30 \mathrm{~s}$ by ion milling in argon, and a $30 \mathrm{~nm}$ Ti layer is deposited immediately before Au sputtering. Thicker Ti adhesion layers affect the R(T) curve of the NSMO sensor, typically apparent as an increased resistance at low temperature that is likely due to an oxygen-depleted NSMO surface layer where the Ti forms $\mathrm{TiO}_{2}$. Thinner Ti layers lead to unreliable Au adhesion, with parts of the Au wiring delaminating from the Si chip during lift-off.

These NSMO sensors show a metal-insulator transition with a resistance peak at $\sim 140$ to $\sim 150 \mathrm{~K}$ (figure 6 ). The optimum temperature for bolometer operation is around $\sim 100$ to $\sim 110 \mathrm{~K}$ where the sensitivity, measured by the temperature coefficient of resistance TCR $\equiv 1 / \mathrm{R} \cdot \partial \mathrm{R} / \partial \mathrm{T}$, reaches its maximum value of up to $13 \% / \mathrm{K}$. Note that the square resistance of these film is rather high due to the oxygen deficiency in the films. While enabling operation at $\sim 100 \mathrm{~K}$, this also leads to increased $1 / \mathrm{f}$ noise [10] and reduces the signal-to-noise ratio of the sensor [4].

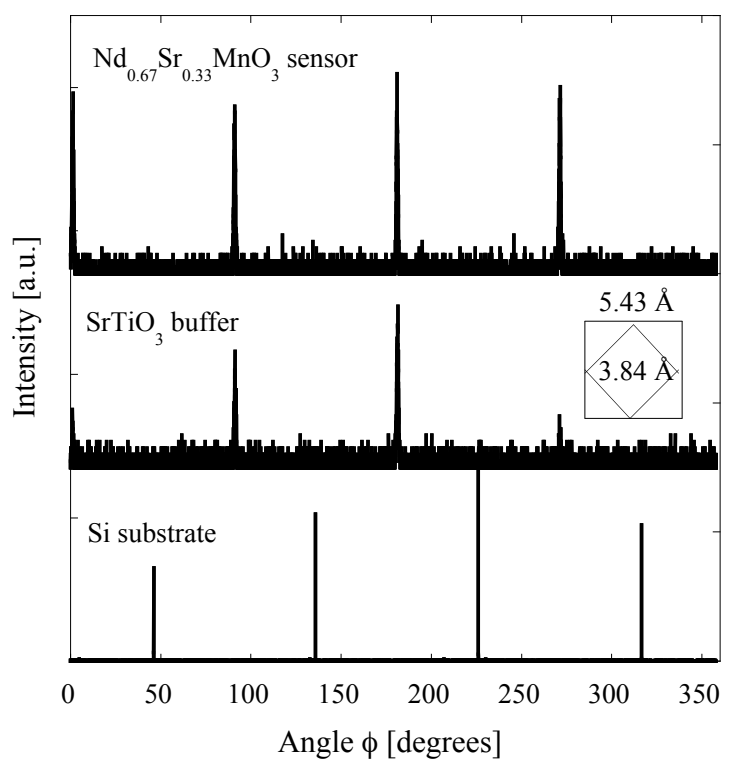

Fig. 5. The diffraction pattern for the three layers demonstrates that the NSMO film is epitaxial on top of the STO-buffered Si [8]. The inset illustrates how STO and NSMO with lattice constants of $3.884 \AA$ and $3.812 \AA$, respectively, can be grown epitaxially on $\mathrm{Si}$ with a lattice constant of $5.43 \AA$.

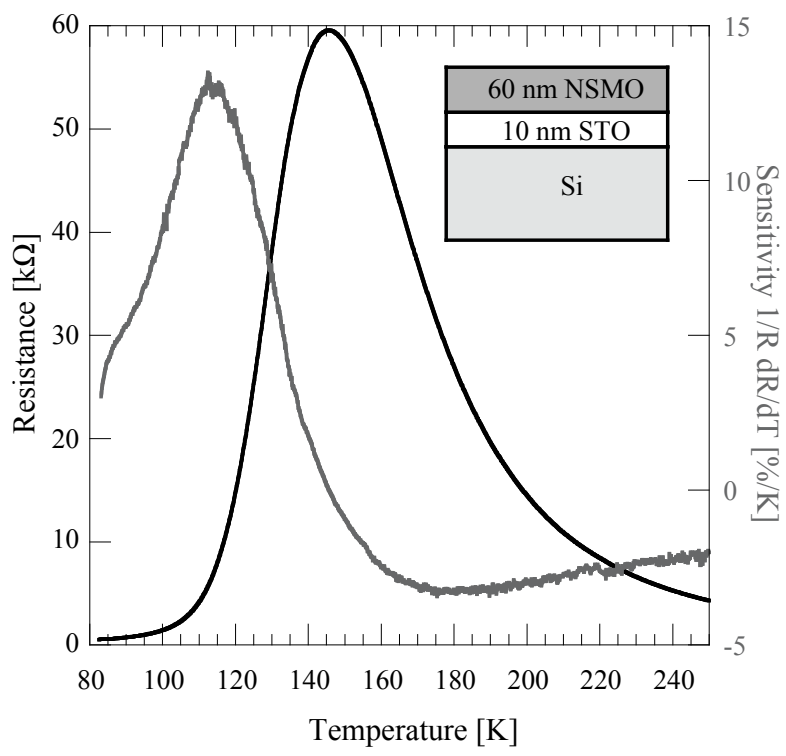

Fig. 6. Resistance and sensitivity, given by the temperature coefficient of resistance $1 / \mathrm{R} \partial \mathrm{R} / \partial \mathrm{T}$, as a function of temperature for an NSMO film on STO-buffered Si. The ideal operating temperature with maximum sensitivity is around $\sim 110 \mathrm{~K}$.

\section{B. Device Response}

The CMR sensors are clamped to an oxygen-free highconductivity (OFHC) copper sample stage, with indium between the $\mathrm{Si}$ chip and the OFHC $\mathrm{Cu}$ for good thermal conductivity across the interface. The sample stage is attached to a liquid-cryogen-free pulse tube refrigerator, mounted inside a vacuum chamber at a pressure of $\sim 10^{-6}$ Torr, and temperature regulated at $\mathrm{T}=110 \mathrm{~K}$. For calibration the sensors are illuminated at a rate of $10 \mathrm{~Hz}$ with a pulsed Nd-YAG laser that has been frequency-doubled to a wavelength of $532 \mathrm{~nm}$ [11]. Its output can be adjusted between $20 \mathrm{~nJ}$ and $12 \mathrm{~mJ}$ per 5 ns pulse to cover the energy range of interest at LCLS.

The response of the total energy monitor at $\mathrm{T}=110 \mathrm{~K}$ to pulsed optical laser light is shown in figure 7. In addition to the thermal signal expected according to figures 1 and 2, a significantly larger prompt signal is observed that overlaps with the thermal signal and affects a measurement of the thermal response. Surprisingly, this prompt signal is observed even at $\mathrm{V}_{\text {bias }}=0$ where any thermal signal is expected to be zero [4]. In addition, while the amplitude of the prompt signal changes with $\mathrm{V}_{\text {bias }}$, it does not increase linearly with $\mathrm{V}_{\text {bias }}$, and it increases roughly logarithmically in some devices with pulse energy, neither of which is expected for thermal devices. The prompt signal does in fact not depend on the presence of an NSMO sensor at all, i.e. it is not of a thermal origin. For these reasons, it cannot be used as an alternative measure of the pulse energy. The prompt signal can change polarity, sometimes as function of temperature or as a function of absorption location, and its magnitude is often irreproducible between nominally identical devices. Control experiments on metallic aluminum samples do not exhibit any fast transients, suggesting that the prompt signals are fundamentally related to the characteristics of the semiconducting absorber material. 


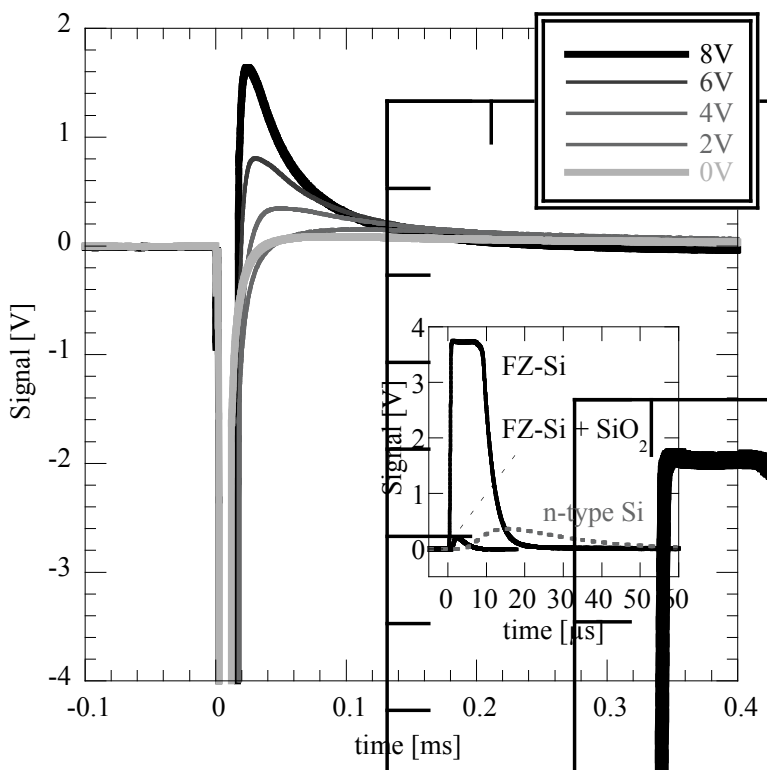

Fig. 7. Photoresponse of the bolometer of figure 6 at $\mathrm{T}=106 \mathrm{~K}$ for different bias voltages. Note that there is a strong prompt signal at $\mathrm{V}=0$, and that the amplitude does not scale linearly with bias. The inset shows the response to $20 \mathrm{~nJ}$ pulses for test devices consisting of just an Au bond pad (no NSMO sensor at all) on float-zone (FZ) and doped (n-type) Si substrates. The response of the FZ-Si device exceeds the preamplifier range of $\mathrm{V}_{\max } \approx 3.8 \mathrm{~V}$.

We have fabricated several test structures without NSMO films to investigate the origin of the prompt transients. These test structures typically consisted of a Si substrate (p-type, ntype, or high-purity float zone $\mathrm{Si}$ ), sometimes with a buffer layer (STO, $\mathrm{SiO}_{2}$ or yttrium-stabilized zirconia YSZ), and a single $\sim 1 \mathrm{~mm}^{2} \mathrm{Au}$ bond pad from where the connection to the input of the preamplifier was made. Initially, the change of polarity in the earliest sensors at $\mathrm{T} \approx 120 \mathrm{~K}$ pointed towards a possible piezoelectric origin, since STO is piezoelectric and the thermal expansion coefficient of $\mathrm{Si}$ changes polarity at $\sim 120 \mathrm{~K}$. A piezoelectric origin of the prompt signal was eventually ruled out for several reasons:

- The presence of the prompt signal does not depend on the presence of an STO buffer film, and Si by itself is not piezoelectric. The prompt signal does not exhibit a polarity change at $120 \mathrm{~K}$ in all Si devices.

- The magnitude of the prompt signal is suppressed by several orders of magnitude when piezoelectric $\mathrm{SiO}_{2}$ is placed between the $\mathrm{Si}$ and the $\mathrm{Au}$ bond pad. The inset in figure 7 shows that the prompt signal in response to the minimum laser energy of $20 \mathrm{~nJ} /$ pulse already saturates the preamplifier output in a high-purity float-zone Si chip, while a test device from the same Si chip with an additional $\mathrm{SiO}_{2}$ layer tested during the same run under identical conditions showed a much smaller prompt signal.

- Test structures with thin YSZ buffers consistently showed a larger prompt signal that those with thick YSZ.

These observations point towards an electron-mediated origin of the prompt signal, albeit one that must be consistent with transient observations at $\mathrm{V}_{\text {bias }}=0$. It is unlikely that the formation of a pn-junction in the $\mathrm{Si}$ and charge separation due to the intrinsic field of the associated depletion layer can fully account for the prompt signal:

- The prompt transients are observed in test structures on highly-doped Si where the depletion layer is at most a few microns thick and located on the opposite site of the laser impact. Electron-hole pairs generated by the absorption of the $532 \mathrm{~nm}$ laser within a few $\mu \mathrm{m}$ of the Si surface will not experience any field until they have diffused into the space charge region.

- While the prompt signal is faster in high-purity float-zone (FZ) Si than in doped Si (inset figure 7), it is not necessarily bigger, although the space charge region should be much larger and the recombination time much longer in FZ $\mathrm{Si}$ and should therefore capture more electron hole pairs before recombination.

We believe that there are two mechanisms that likely account for the observed prompt transients. One is due to the different mobilities of electron and holes in $\mathrm{Si}$, which causes them to separate during diffusion along the concentration gradient in the $\mathrm{Si}$ absorber after non-uniform illumination (Dember effect) $[12,13]$. Since electron mobilities are higher than hole mobilities in $\mathrm{Si}$, the laser-absorbing surface charges up positively, generating an observable voltage until the electrons and holes either recombine or are distributed evenly throughout the $\mathrm{Si}$. This process depends not only on the relative mobilities of the electrons and holes in $\mathrm{Si}$, but also on the relative velocity of their recombination at surface states. This can lead to a polarity reversal of the Dember voltage when front-surface recombination of the holes is faster than the back-surface recombination [13]. This depends on details of the surface treatment and therefore varies sensitively with sensor preparation. The magnitude of the Dember effect is non-linear with laser energy since a high density of electronhole pairs can produce a Dember voltage large enough to drag holes along with the faster electrons, and thus prevent charge separation until the electron-hole density (and thus the Dember voltage) has decreased significantly from its initial value. Insulators, including piezoelectric buffers, reduce the coupling between the Dember signal and the Au bond pad.

The second effect responsible for fast transients is consistent with the diffusion of thermally generated majority carriers in the Si substrate from higher to lower temperature regions (transient thermoelectric, or Seebeck effect) $[12,14]$. The polarity of this effect depends on the polarity of the majority carriers in the $\mathrm{Si}$, i.e. positive for $\mathrm{n}$-type and negative for p-type doping. The transient thermoelectric signal decays with a characteristic carrier recombination time or the time for temperature equilibration within the $\mathrm{Si}$ chip, whichever is shorter.

\section{BOLOMETERS FOR $180 \mathrm{~K}$ OPERATION}

\section{A. CMR Sensor fabrication}

The easiest way to address the interference of the prompt transients with the thermal response of the NSMO sensor is to slow down the thermal signal such that it peaks at a time when the two prompt signals have decayed to zero. To avoid having to slow down the thermal signal to the point that it is no longer 


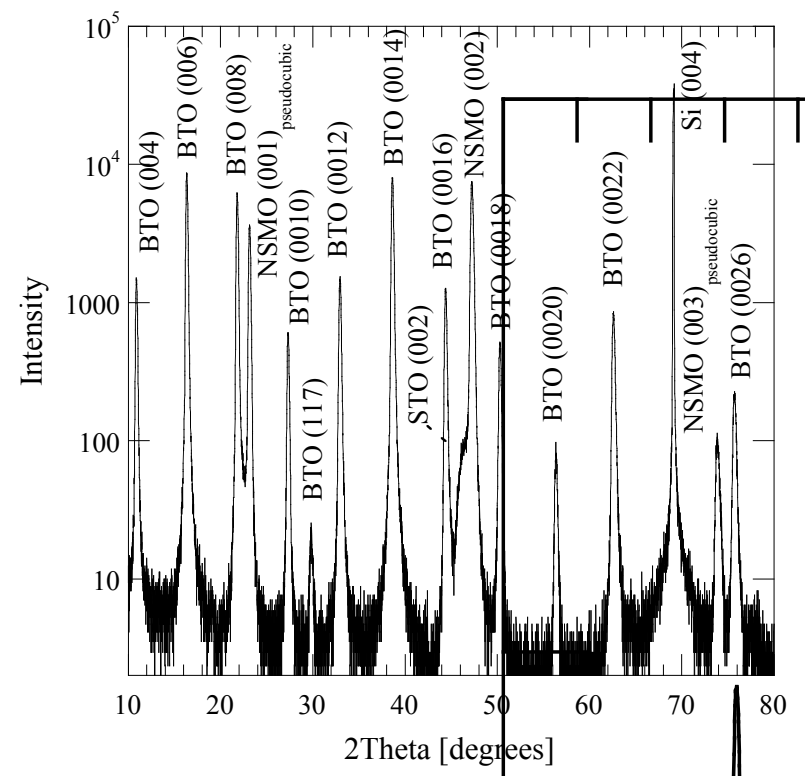

Fig 8. The diffraction pattern for the four layers demonstrates that the NSMO film is epitaxial on top of the BTO-STO-buffered $\mathrm{Si}$ [8].

compatible with the LCLS repeat frequency, it is helpful to speed up the prompt transients as much as possible. This is accomplished using high-purity float-zone $\mathrm{Si}$ with a resistivity $>10 \mathrm{k} \Omega \mathrm{cm}$ as a starting material (see inset figure 7). We also introduce a secondary bismuth titanate (BTO) buffer layer on top of the STO-buffered $\mathrm{Si}$ to reduce the lattice mismatch between the buffer and the NSMO film. The BTO is deposited by pulsed laser deposition, and can be grown epitaxially on STO (figure 8). This secondary buffer reduces the strain in the NSMO, which is deposited by PLD at increased $790{ }^{\circ} \mathrm{C}$ and cooled down at a higher oxygen pressure of 500 Torr. This produces sensor films that are not oxygen-deficient and whose metal-insulator transition peaks at a temperature of $\sim 200 \mathrm{~K}$, closer to the temperature of bulk NSMO (figure 9) [6].

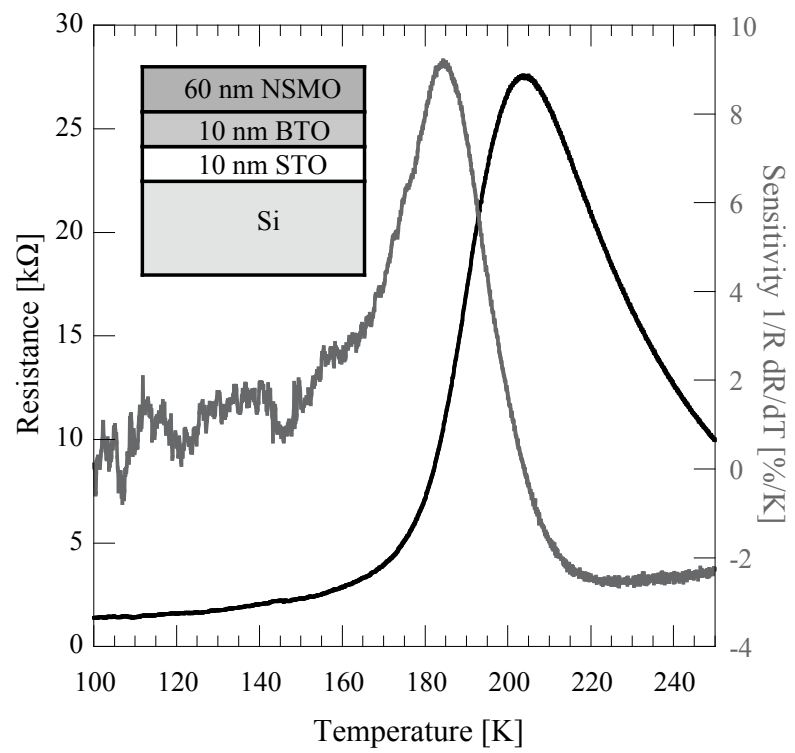

Fig 9. R(T) and TCR of NSMO on STO-BTO-buffered Si for total energy monitor operation at the temperature $\mathrm{T}=185 \mathrm{~K}$ with maximum TCR.

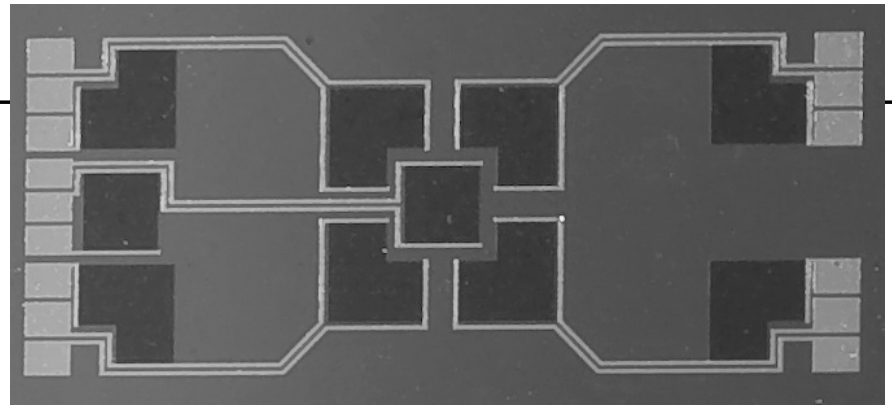

Fig. 10. Photograph of a five-pixel bolometer device. The central pixel (dark) is surrounded by four L-shaped pixels for spatial resolution. Each pixel has a matching partner along the edge of the chip that will not be illuminated by the LCLS beam but serve as a bridge completion resistor in the bridge readout.

In addition, these NSMO films have a lower resistivity and less $1 / \mathrm{f}$ noise, which increases the signal-to-noise ratio for total energy measurements at LCLS.

As before, the sensor areas are defined by photolithography with a $\mathrm{KI} / 10 \% \mathrm{HCl}$ etch. The $\mathrm{Au}$ wiring layers and bond pads as well as the $30 \mathrm{~nm}$ Ti adhesion layer are deposited by magnetron sputtering, and their geometry is defined by lift-off. Single-pixel and five-pixel devices have been fabricated, all with matching resistors on the chip to complete the bridge circuit of the pre-amplifier at low temperature for good common-mode noise rejection (figure 10). "Island"-type devices (figures 3c and 3d) are fabricated by subsequent deep reactive ion etching (DRIE) of the Si with $\mathrm{SF}_{6}$.

\section{B. Sensor response}

The sensors are mounted on the OFHC $\mathrm{Cu}$ sample holder inside the vacuum chamber, cooled and temperature-regulated at the operating temperature of $185 \mathrm{~K}$ where they exhibit the highest sensitivity (figure 9), and illuminated by the pulsed optical laser with an energy from $20 \mathrm{~nJ}$ to $2 \mathrm{~mJ}$ per $5 \mathrm{~ns}$ pulse.

Figure 11 shows the response of the sensor to $1 \mathrm{~mJ}$ pulses of the $532 \mathrm{~nm}$ calibration laser. As expected according to figure 1 , the thermal signal is greatly slowed down compared to sensors operated at $\mathrm{T}=110 \mathrm{~K}$, with the thermal signal reaching its maximum value only after $\mathrm{t} \approx 250 \mu \mathrm{s}$. In addition to the thermal signal, the two prompt transients are clearly visible. The Dember voltage (a) is very fast in the high-purity $\mathrm{Si}$ absorber and sufficiently strong to saturate the preamplifier output. The preamplifier has a recovery time of $\sim 5 \mathrm{~ns}$, and is therefore fast enough to record the somewhat slower transient thermoelectric signal (b) that peaks after $\sim 40 \mu \mathrm{s}$. Most importantly, both prompt transients have fully decayed to zero by the time the thermal signal reaches its maximum. The amplitude of the thermal response at $t=250 \mu \mathrm{s}$ is therefore linear with the incident laser energy over the entire energy range of interest for LCLS (inset figure 11).

Note that the signal shape of the thermal signal agrees very well with the finite element calculations for short time scales (dashed line). However, for longer time scales the signal does not yet decay as quickly as predicted. After $100 \mathrm{~ms}$, the thermal signal has only decayed to $\sim 3 \%$ of its peak value, indicating that the thermal coupling of the $\mathrm{Si}$ chip to the OFHC sample holder through the In contact should be 


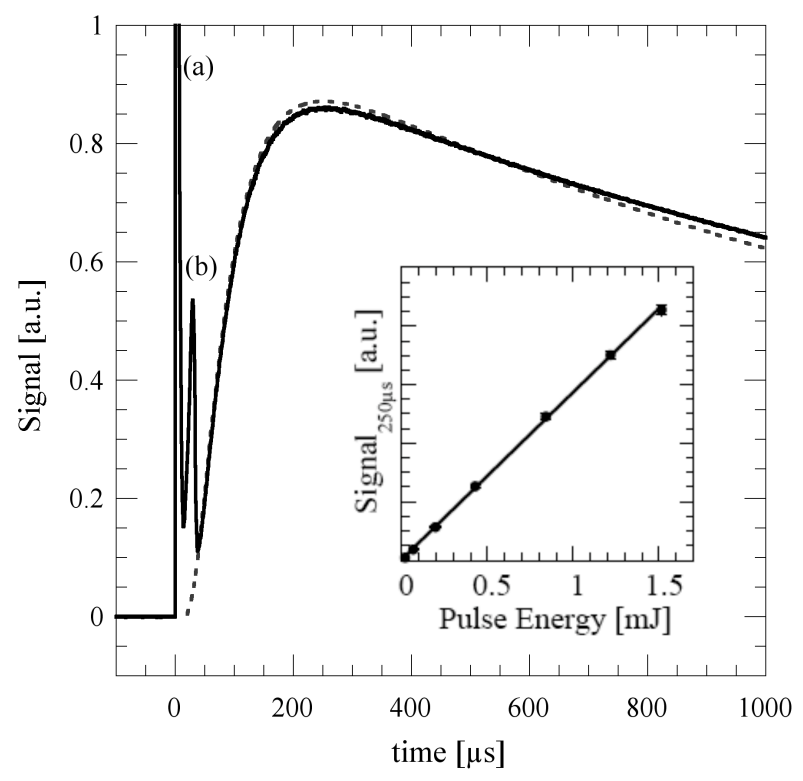

Fig. 11. Photoresponse of the thermal sensor of figure $9 f$ at $\mathrm{T}=185 \mathrm{~K}$ at a bias voltage of $4 \mathrm{~V}$. Note that the prompt signals, i.e. the Dember voltage (a) and the transient thermoelectric voltage (b), have decayed by the time the thermal signal reaches its peak. The pulse shape of the measured thermal signal (solid line) is in good agreement with the calculations (dashed line).

improved for LCLS operation at $10 \mathrm{~Hz}$ during commissioning. Also, the amplitude of the thermal signal is about a factor 4 smaller than expected. This is likely due to the formation of a diode-like structure at the interface between the sensor and the buffer layer, and the subsequent partial shunting of the signal through the substrate. We have measured the signal at $180 \mathrm{~K}$ at different bias voltages, and observe a bias-dependent decrease in CMR resistance and in output signal that can be modeled as a sensor shunted by a small diode. These unexpected observations, however, do not prevent the use of NSMO sensors on buffered $\mathrm{Si}$ as total energy monitors at LCLS.

\section{SUMMARY AND OUTLOOK}

We have fabricated neodymium strontium manganese oxide $\left(\mathrm{Nd}_{0.76} \mathrm{Sr}_{0.33} \mathrm{MnO}_{3}\right.$, or NSMO) sensors on buffered $\mathrm{Si}$ absorbers for thermal total energy measurements of the LCLS free electron X-ray laser. Epitaxial NSMO films with a high temperature coefficient of resistance (TCR) $>10 \% / \mathrm{K}$ have been successfully deposited on Si using STO and BTO buffer layers. The $\mathrm{Si}$ is expected to be radiation hard even for illumination at free electron lasers such as LCLS [5], and the sensor has sufficient sensitivity and linearity to cover the energy range up to $2 \mathrm{~mJ} /$ pulse at LCLS. When operated at a temperature of $180 \mathrm{~K}$, the sensor rise time is sufficiently slow to avoid being affected by prompt transients. Better sensor coupling to the $\mathrm{Cu}$ sample holder will accelerate the cooldown to be compatible with LCLS operation at $10 \mathrm{~Hz}$ during commissioning.

As a thermal sensor, the response of the total energy monitor is expected to suffer from the least unknowns in the ultra-short X-ray pulses at LCLS. For example, it can be absolutely calibrated with a comparably "slow" $5 \mathrm{~ns}$ pulsed optical laser, because the thermal response of the sensor is orders of magnitude slower still, and most of the energy for both LCLS and the calibration laser will be ultimately converted into heat. This thermal total energy monitor will therefore mostly be used to calibrate secondary pulse energy meters such as scintillators or gas detectors during LCLS commissioning.

If desired, it is possible to further improve the sensor performance in future upgrades of the LCLS diagnostics. The $\mathrm{Ti}$ adhesion layer below the Au wiring can be replaced by $\mathrm{Cr}$ to reduce the extent to which the NSMO surface is degraded by oxygen removal. Alternative buffers layer such as $\mathrm{CeO}_{2}$ can be explored to remove the partial shunt of the sensor through the substrate and increase the signal amplitude. Radiation damage tests of the NSMO sensor material are currently in progress.

\section{REFERENCES}

[1] H. Winick et al., "Short wavelength FELS using the SLAC linac", Nucl. Inst. Meth. A, vol. 347, pp. 199 - 205, 1994

[2] J. Andruszkow et al.," First Observation of Self-Amplified Spontaneous Emission in a Free-Electron Laser at $109 \mathrm{~nm}$ Wavelength" Phys. Rev. Lett., vol. 85, pp. 3825 - 3829, 2000

[3] A. Wootton, J. Arthur, T. Barbee, R. Bionta, A. Jankowski, R. London, D. Ryutov, R. Shepherd, V. Shlyaptsev, R. Tatchyn, A. Toor, "Research and development for X-ray optics and diagnostics on the linac coherent light source (LCLS)", Nucl Inst. Meth. A, vol. 483, pp. 345 - 350, 2002

[4] S. Friedrich, L. Li, L. L. Ott, R. M. Kolagani, G. J. Yong, Z. A. Ali, O. B. Drury, E. Ables, R. M. Bionta, "Design of a bolometer for total energy measurement of the Linear Coherent Light Source pulsed X-ray laser", Nucl. Inst. Meth. A, vol. 559, pp. 772 - 774, 2006

[5] S. P. Hau-Riege et al., "Damage threshold of inorganic solids under freeelectron-laser irradiation at $32.5 \mathrm{~nm}$ wavelength", Appl. Phys. Lett., vol. 90, pp. 173128 - 173130, 2007.

[6] A. Goyal, M. Rajeswari, R. Shreekala, S. E. Lofland, S. M. Bhagat, T. Boettcher, C. Kwon, R. Ramesh, T. Venkatesan, "Material characteristics of perovskite manganese oxide thin films for bolometric applications", Appl. Phys. Lett., vol. 71, pp. 2535 - 2537, 1997

[7] M. Rajeswari, C. H. Chen, A. Goyal, C. Kwon, M. C. Robson, R. Ramesh, T. Venkatesan," Low-frequency optical response in epitaxial thin films of $\mathrm{La}_{0.67} \mathrm{Ca}_{0.33} \mathrm{MnO}_{3}$ exhibiting colossal magnetoresistance" Appl. Phys. Lett., vol. 68, pp. 3555 - 3557, 1996

[8] Y. Liang, Y. Wei, X. M. Hu, Z. Yu, R. Droopad, H. Li, K. Moore, "Heteroepitaxy of $\mathrm{SrTiO}_{3}$ on vicinal $\mathrm{Si}(001)$ : Growth and kinetic effects", J. Appl. Phys., vol. 96, pp. 3413 - 3416, 2004

[9] G. C. Yong, R. M. Kolagani, D. Cox, R. Mundle, A. Davidson III, S. Friedrich, Z. Ali, O. Drury, L. Li, L. L. Ott, Y. Liang, "Development of CMR Manganite Sensors for Energy Measurements of the Linear Coherent Light Source Free Electron Laser X-ray Pulses", Materials Research Society 2006 Spring Meeting April 17-21 San Francisco, CA Published on-line at http://www.mrs.org/ 2006 Spring Proceedings Symposium GG, vol. 928E, paper \#: 0928-GG05-05; and G. C. Yong et al., in preparation

[10] M. Rajeswari, R. Shreekala, A. Goyal, S. E. Lofland, S. M. Bhagat, K. Ghosh, R. P. Sharma, R. L. Greene, R. Ramesh, T. Venkatesan, "Correlation between magnetic homogeneity, oxygen content, and electrical and magnetic properties of perovskite manganite thin films", Appl. Phys. Lett, vol. 73, pp. 2672 - 2674, 1998

[11] Minilite-1 Nd-YAG laser, manufactured by Continuum Inc.

[12] W. Hahn, M. Boshta, K. Bärner, R. Braunstein, "The charge transport properties of a-Si:H thin films under hydrostatic pressure", Mat. Sci. Eng. B, vol. 130, pp. $184-186,2006$

[13] S. K. Chattopathyaya, V. K. Mathur, "Normal and anomalous Dember effect", J. Appl. Phys., vol. 40, pp. 1930 - 1933, 1969

[14] M. Sasaki, H. Negishi, M. Inoue, "Pulsed laser-induced transient thermoelectric effects in silicon crystals", J. Appl. Phys., vol. 59, pp. 796 - 802, 1986 